\title{
The Efficacy of Fibroblast Growth Factor for the Treatment of Chronic Vocal Fold Scarring: From Animal Model to Clinical Application
}

\author{
Myung Jin Ban ${ }^{1} \cdot$ Jae Hong Park $\cdot$ Jae Wook Kim² $\cdot$ Ki Nam Park ${ }^{3} \cdot$ Jae Yong Lee ${ }^{3} \cdot$ Hee Kyung Kim ${ }^{4} \cdot$ Seung Won Lee ${ }^{3}$ \\ ${ }^{1}$ Department of Otolaryngology-Head and Neck Surgery, Soonchunhyang University College of Medicine, Cheonan; ${ }^{2}$ Department of \\ Otolaryngology-Head and Neck Surgery, Soonchunhyang University College of Medicine, Seoul; Departments of ${ }^{3}$ Otolaryngology-Head and \\ Neck Surgery and ${ }^{4}$ Pathology, Soonchunhyang University College of Medicine, Bucheon, Korea
}

Objectives. This study assessed the regenerative efficacy of basic fibroblast growth factor (FGF) in a rabbit model of chronic vocal fold scarring and then confirmed its utility and safety in a prospective trial of patients with this condition.

Methods. FGF was injected three times, at 1-week intervals, into a chronic vocal fold scar created in a rabbit model.After 1 month, mRNA level of procollagen I, hyaluronic acid synthetase 2 (HAS 2), and matrix metalloproteinase 2 (MMP 2) were analyzed by real-time polymerase chain reaction. The relative densities of hyaluronic acid (HA) and collagen were examined 3 months post-injection. From April 2012 to September 2014, a prospective clinical trial was conducted at a tertiary hospital in Korea. FGF was injected into the mild vocal fold scar of 17 consecutive patients with a small glottic gap. The patients underwent perceptual, stroboscopic, acoustic aerodynamic test, and Voice Handicap Index (VHI) survey prior to and 3, 6, and 12 months after FGF injection.

Results. FGF injection of the vocal fold scar decreased the density of collagen and increased mRNA level of HAS 2 and MMP 2 expression significantly compared to the control group injected with phosphate buffered solution in a rabbit model $(P<0.05)$. In the clinical trial, significant improvements in the majority of the subjective and objective voice parameters were registered 3 months after FGF injection and were maintained at 12 months. Complications associated with the FGF injections, such as granuloma, were not observed during the follow-up period.

Conclusion. Based on the animal model and the prospective clinical trial, vocal fold injections of FGF in patients with mild chronic vocal fold scarring can significantly improve voice quality for as long as 1 year and without side effects. Our results recommend the use of FGF vocal fold injection as an alternative treatment modality for mild chronic vocal fold scarring.

Keywords. Fibroblast Growth Factors; Vocal Cord; Dysphonia

\section{INTRODUCTION}

The vocal fold, with its highly differentiated, multilayer struc-

- Received July 15, 2016

Revised August 25, 2016

Accepted August 25, 2016

- Corresponding author: Seung Won Lee

Department of Otolaryngology-Head and Neck Surgery, Soonchunhyang

University Bucheon Hospital, Soonchunhyang University College of

Medicine, 170 Jomaru-ro, Bucheon 14584, Korea

Tel: +82-32-621-5450, Fax: +82-32-621-5016

E-mail: 1sw0922@schmc.ac.kr ture, can be damaged by trauma, surgery, drugs, or radiation. The resulting scarring deforms the fold and inhibits vocal vibratory function. Both conservative and surgical treatment fail to resolve voice impairment, such that chronic vocal fold scarring is considered incurable. The number of patients with voice problems is increasing and includes those with chronic vocal fold scarring after voice surgery. For people who rely on their voice for their occupation, such as teachers, singers, clergy, and telemarketers, chronic vocal fold scarring affects not only their health but also their economic security [1].

Fibroblast growth factor (FGF), a member of the larger FGF

Copyright @ 2017 by Korean Society of Otorhinolaryngology-Head and Neck Surgery.

This is an open-access article distributed under the terms of the Creative Commons Attribution Non-Commercial License (http://creativecommons.org/licenses/by-nc/4.0)

which permits unrestricted non-commercial use, distribution, and reproduction in any medium, provided the original work is properly cited. 
family, plays a crucial role in facilitating vocal fold regeneration. FGF inhibits granuloma formation, and promotes scarless tissue repair, by inhibiting collagen and extracellular matrix $(\mathrm{ECM})$ deposition and hyaluronic acid (HA) generation in lamina propria [2]. Previously reported surgical approaches, such as resection and laser ablation of the vocal fold scar, showed limited results due to an unpredictable wound healing process and regeneration of the lamina propria. The use of growth factors, including FGF, to induce vocal fold regeneration is regarded as one of the most effective alternative treatments in improving the regeneration of the vocal fold. Therefore, in this study we tested the utility and safety of FGF injection to treat chronic vocal fold scarring in an animal model. After determining that FGF was effective in vocal fold regeneration, we carried out a prospective clinical trial in patients with vocal fold scarring (Fig. 1).

\section{MATERIALS AND METHODS}

\section{Ethics statement}

After the approval of this study by the Animal Ethics Committee, animal was cared strictly in accordance with institutional guidelines. All procedure was conducted under anesthesia by xylazine $(5 \mathrm{mg} / \mathrm{kg})$ with an intramuscular injection of $50 \mathrm{mg} / \mathrm{kg}$ of ketamine hydrochloride to minimize suffering. Prospective human clinical trial was conducted following Soonchunhyang University Bucheon Hospital Institutional Review Board approval (IRB No.: SCHBC_IRB_ 2010_ 85). The authors registered this study after enrolment of participants because of the ownership of this novel treatment. The use of FGF in laryngology and plastic surgery has already commercialized and this study is not related to any patent. All patients agreed to written informed consent before surgery. The authors confirm that the trial for this intervention are registered.

\section{Animal model of chronic vocal fold scar and FGF injection}

This 2-year animal model study was carried out in 24 adult laboratory-conditioned rabbits (mean body weight: $2.8 \mathrm{~kg}$ ) with chronic vocal cord scarring similar as previous study [3]. Animal care and surgery were performed according to the policies of Soonchunhyang University Bucheon Hospital. The Institutional

\section{H I G G H L I I G H T T S}

- Fibroblast growth factor (FGF) injections are an alternative treatment modality for chronic vocal fold scarring.

- FGF fold injections in patients with vocal fold scarring can improve voice quality.

- FGF injection decreased the density of collagen and increased hyaluronic acid synthetase 2 and matrix metalloproteinase 2 expression.

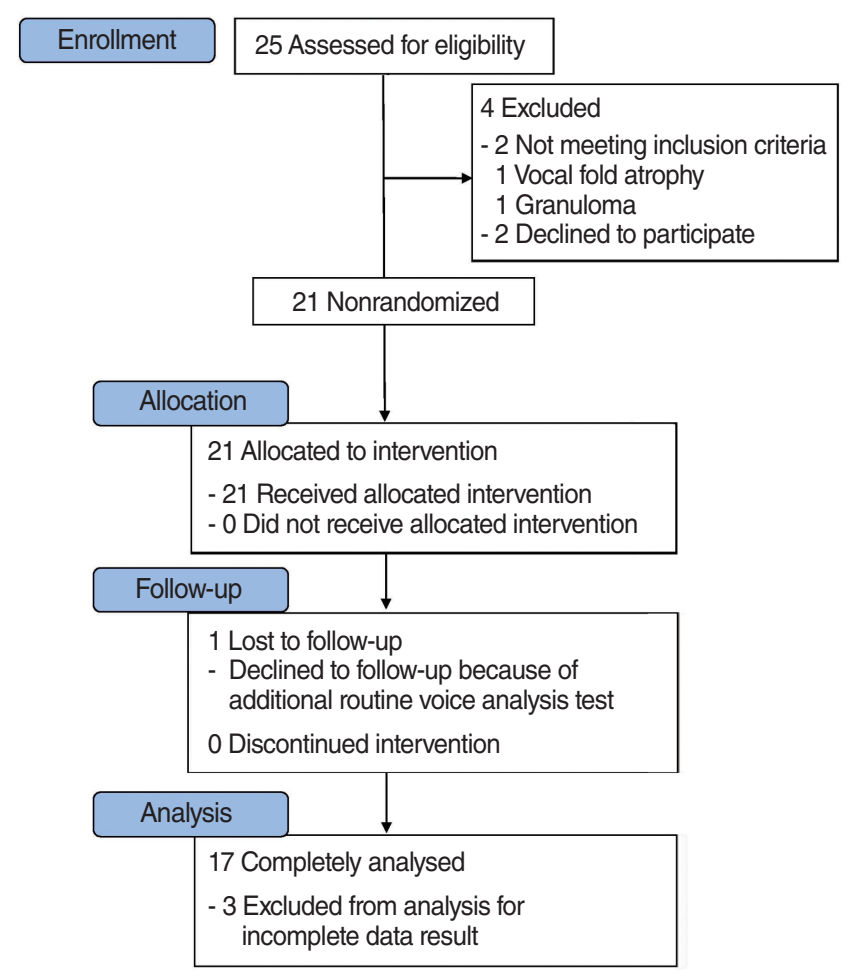

Fig. 1. Consort flow diagram. A common reason for declining trial participation at enrollment and follow-up was that long travel distances made patients unwilling to return to the hospital for the 1-year follow-up visit. Chronic vocal fold scar is a clinical diagnosis, and 2 patients were found at stroboscopy to have vocal fold disease where fibroblast growth factor injection is not indicated. Three patients were excluded for incomplete voice evaluation data result.

Review Board (IRB No.: SCHBC_Animal_201010) approved the experimental use of the animals. Single housing in the cage was used to limit the environmental factor. Dried pelleted diets based on hay was fed at around $60-80 \mathrm{~g} / \mathrm{kg} /$ day. Daily observations and weekly health checks were carried out by animal careers. Two weeks after making vocal fold scar, 2 rabbits died because of stress induced pathologic grooming and hairball induced gastrointestinal problems prior to the experimental endpoint. Twenty four rabbits were raised well and analysed for this study. Ill rabbits were identified by specific abnormal behaviors and they were provided with high standards of care by special animal manager. During the experiment, we had a protocol in place for the early euthanasia/humane endpoints for animals; weight loss of $20 \%-25 \%$, anorexia up to $5-7$ days, weakness to obtain feed, severe infection, signs of severe organ system dysfunction (respiratory, cardiovascular, gastrointestinal: severe vomiting or diarrhea, urogenital, nervous, musculoskeletal, integumentary). Sodium pentobarbital $\geq 100 \mathrm{mg} / \mathrm{kg}$ IP (intraperitoneal injection) was used for rabbit euthanasia.

After anesthesia, the vocal folds were exposed through the oral cavity using a long nasal speculum. A $30^{\circ}, 2.7 \mathrm{~mm} \times 30 \mathrm{~cm}$ telescope (Karl Storz Co., Tuttlingen, Germany) maximized vi- 
sualization of the region of interest through the speculum. The epithelial layer and lamina propria were then separated from the vocalis muscle on both sides of the vocal fold using a laryngeal microsurgery device (upward microscissor; Microfrance, Medtronic Co., Saint Aubin le Monial, France). Scar maturation was complete 3 months after vocal fold scarring surgery. All chronic vocal fold scarring procedures were performed by the same surgeon (SWL) to reduce procedure bias.

With the rabbits under anesthesia as described above, they were divided into an experimental group and a control group. The each side of lamina propria of vocal fold was injected weekly with $0.1 \mathrm{~mL}(10 \mu \mathrm{g})$ of FGF (Fiblast, Kaken Pharmaceutical Co., Tokyo, Japan) using a 27-gauge Xomed orotracheal laryngeal injector (Medtronic, Minneapolis, MN, USA) for three times (experimental group, $n=12$ ). Phosphate-buffered saline (PBS) was injected in each side of vocal fold in the same way (control group, $n=12$ ). Power calculations have been done using our data based on 2,000 Monte Carlo samples from the null distributions by using PASS 12 (NCSS, Kaysville, UT, USA) program. When there is a difference at the 0.05 significance level using a two-sided Mann-Whitney Wilcoxon test, group sample sizes of 6 and 6 achieve $83 \%$ (relative density of HA), 100\% (relative density of collagen), 100\% (procollagen), 89\% (hyaluronic acid synthetase 2 [HAS 2]), and 100\% (matrix metalloproteinase 2 [MMP 2]) power to show a mean difference. The rabbits were euthanized for laryngeal harvest to investigate the mRNA level of ECM gene and histology of vocal fold each at 1 month and 3 months after 3 times of injections. The sequential regenerative ability of FGF was evaluated. Based on the lifespan of rabbits, 3 months was considered long enough to evaluate the long term effect of FGF injection.

\section{Real-time PCR of ECM gene expression in the vocal fold}

The laryngeal tissues of the twelve rabbits were used to analyze ECM gene expression by real-time polymerase chain reaction (PCR). After dissection of the vocal folds from the larynx, the homogenized tissues in a Mixer Mill (Retsch Inc., Pittsburgh, PA, USA) were made. The isolated total RNA produced by an RNeasy kit (Qiagen, Valencia, CA, USA) was prepared. High

Table 1. Primer sequences

\begin{tabular}{lll}
\hline Target & \multicolumn{1}{c}{ CDNA primers } \\
\hline $\begin{array}{lll}\text { Procollagen } \\
\text { type I }\end{array}$ & Forward & 5'-CTGCAAGAACAGCATTGCAT-3' \\
& Reverse & 5'-TCAAGGAAGGGAAAACGAGA-3' \\
HAS 2 & Forward & 5'-GGACGAAGCGTGGATTATGT-3' \\
& Reverse & 5'-ATAAGACTGGCAGGCCCTT-3' \\
MMP 2 & Forward & 5'-TGGATCCTCCTACAGCAGCTGCACCAG-3' \\
& Reverse & 5'AAGAATCCCGTAGAGCTCTTGAATGC-3' \\
B-actin & Forward & 5'-GCTATTGGCGCTGGACTT-3' \\
& Reverse & 5'-GCGGCTCGTAGCTCTTCTC-3' \\
\hline
\end{tabular}

HAS, hyaluronic acid synthetase; MMP, matrix metalloproteinase.
Capacity cDNA reverse transcription kit (Applied Biosystems, Foster City, CA, USA) was utilized for reverse transcription.

Rabbit-specific primers for procollagen type I, HAS 2, MMP 2 and $\beta$-actin were purchased from Integrated DNA Technologies (Coralville, IA, USA) based on previously published sequences (Table 1) [4]. Using the iQ SyBR kit (Bio-Rad, Hercules, CA, USA), real-time PCR was conducted. The quantified mRNA levels in the tissue samples were compared. Relative gene expression (fold-change) was tested by the 2- $\Delta \Delta \mathrm{CT}$ method. The housekeeping gene $\beta$-actin was evaluated as an internal control. The data from the FGF $(n=6)$ vs. PBS $(n=6)$ injection group were compared statistically.

\section{Histology of the FGF-injected vocal fold}

The tissues from twelve rabbits were prepared after fixation in $10 \% \mathrm{v} / \mathrm{v}$ formaldehyde and embedding in paraffin. The middle portion of the membranous vocal fold was analysed histologically from the serial laryngeal coronal section ( $6 \mu \mathrm{m}$ thick). The sections were stained with hematoxylin and eosin (HE); HA was detected by alcian blue staining, and collagen by Masson's trichrome staining. The ratio of special stained pixels relative to the whole HE-stained area was determined in both the lamina propria and the vocalis muscle. The ratio was interpreted as the relative density of HA or collagen by one pathologist blinded to the treatment to minimize measurement bias. The same method was used in our previous study of fat block implantation in the vocal fold using a minithyrotomy approach [5]. The data from the FGF $(n=6)$ vs. PBS $(n=6)$ injection group were analyzed statistically.

\section{Prospective human clinical trial of FGF injection in patients with chronic vocal fold scarring \\ Patients}

On the basis of the previous rabbit study, a prospective human clinical trial was conducted following IRB approval (IRB no.: SCHBC_IRB_2010_85). From April 5, 2012 to February 2, 2013, patients with dysphonia due to mild vocal fold scarring and with a minimal glottis gap were recruited. The size of the glottis gap was measured at less than $2 \mathrm{~mm}$ and resulted in decreased vocal vibration seen on stroboscopy. All 17 patients who participated in the study had a history of chronic vocal fold scarring due to previous laryngeal microsurgery, with a history lasting over 1 year. They were followed-up until September 25, 2014 by a single head and neck surgeon. Patients with a history of other vocal fold diseases, such as atrophy, mucosal lesion, granuloma, or sulcus vocalis, or a history of malignant tumors of the vocal fold were excluded.

\section{FGF injection}

A single surgeon (SWL) injected the FGF percutaneously after local anesthesia using transnasal fiberscopic monitoring (Olympus laryngobronchoscope ENF type T3 ver. 2, Olympus, Tokyo, Japan). FGF (Fiblast), at a dose of $20-30 \mu \mathrm{g}$ in $0.2-0.3 \mathrm{~mL}$ re- 
constituted solution, was injected at Reinke's space. The choice of injection approach-cricothyroid, transcartilagenous, or thyrohyoid-depended on the accessibility to the vocal fold (Fig. 2).

\section{Objective and subjective voice analysis}

Voice quality was analysed prior to and 3, 6, and 12 months after FGF injection. The average percentages of jitter and shimmer, as well as the harmonics-to-noise ratio (HNR), are objective voice parameters and calculated with the Multi-Dimensional Voice Program (MDVP; model 4500, Kay Pentax). Another objective parameters for both the maximum phonation time (MPT) and the voice range profile (VRP) were checked using the Computerized Speech Lab (CSL model 4500, Kay Pentax) and averaged after three trials. Auditory perception was measured using the overall sum of the GRABAS (grade, roughness, breathiness, asthenia, strain) scale [6]. Psychosocial data were surveyed using the Voice Handicap Index (VHI-10, Korean language version) [7]. Endoscopic and videostroboscopic results were obtained via a stroboscope (Model 9100, Kay Pentax). Glottal closure and
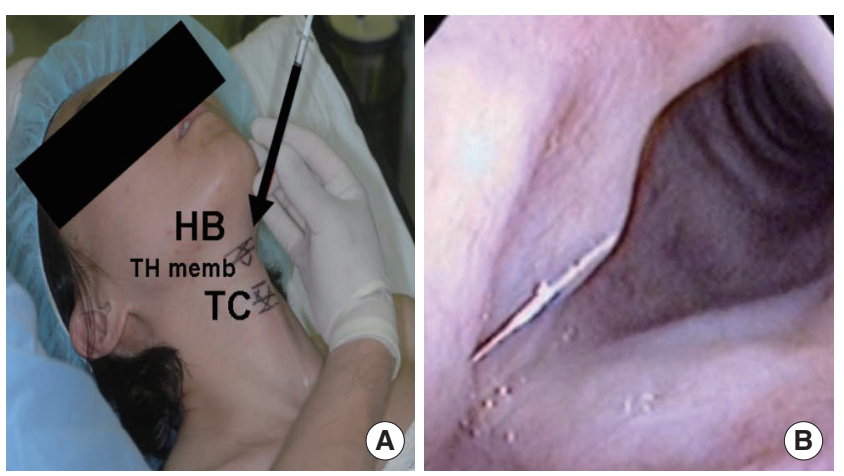

Fig. 2. Injection of fibroblast growth factor into lamina propria of chronic vocal fold under local anesthesia. (A) Surgical photograph of procedure and (B) fiberoscopic view of injection. $\mathrm{HB}$, hyoid bone; $\mathrm{TH}$ memb, thyrohyoid membrane; TC, thyroid cartilage.

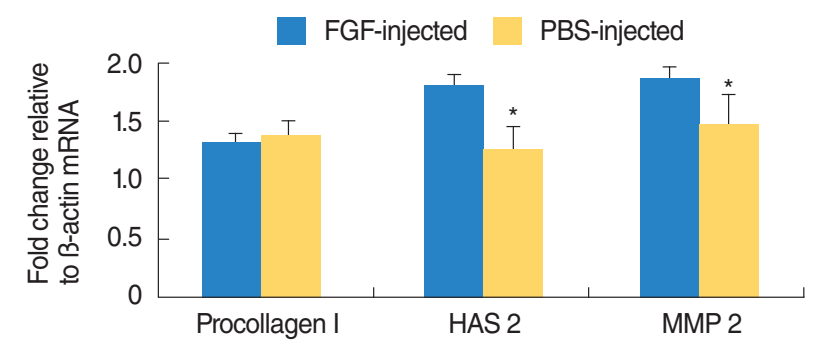

Fig. 3. Normalized mRNA expression ratios of procollagen type I, hyaluronic acid synthase 2 (HAS 2), and matrix metalloproteinase 2 (MMP 2). The results are expressed as the fold change in target gene mRNA expression relative to mRNA expression of the housekeeping gene, B-actin. Each sample was tested in duplicate. HAS 2 and MMP 2 expression increased significantly in the FGF-injected group vs. PBS-injected group. The error bars represent the $95 \%$ confidence intervals of the mean. FGF, fibroblast growth factor; PBS, phosphate-buffered saline. ${ }^{\star} P<0.05$ in a Mann-Whitney U-test. the mucosal wave were evaluated using a previously reported, independent, four-point scale where $0=$ severe glottic gap, no wave; 1 =moderate glottic gap, obvious decreased mucosal wave; 2 =mild glottic gap, slightly decreased mucosal wave; and $3=$ complete closure, full wave [8].

\section{Statistical analyses}

The Wilcoxon signed-rank test and Mann-Whitney $U$-test via the SPSS ver. 17.0 (SPSS Inc., Chicago, IL, USA) were performed. Statistical significance was indicated as $P$-value $<0.05$.

\section{RESULTS}

\section{Real-time PCR of ECM gene expression in the vocal fold}

One month after FGF injection, the fold changes in the mRNA of the target genes relative to the mRNA level of the housekeeping gene $\beta$-actin were determined. A Mann-Whitney $U$-test revealed significant increases in HAS 2 and MMP 2 expression in the FGF vs. the PBS injection group whereas the decrease in procollagen type I expression on the treated group was without statistical significance (Fig. 3).

\section{Histology of the FGF-injected vocal fold in a rabbit model}

Three months after FGF injection, the relative densities of HA and collagen in the FGF-injected and control groups were compared. In the FGF injection group, a representative coronal sec-
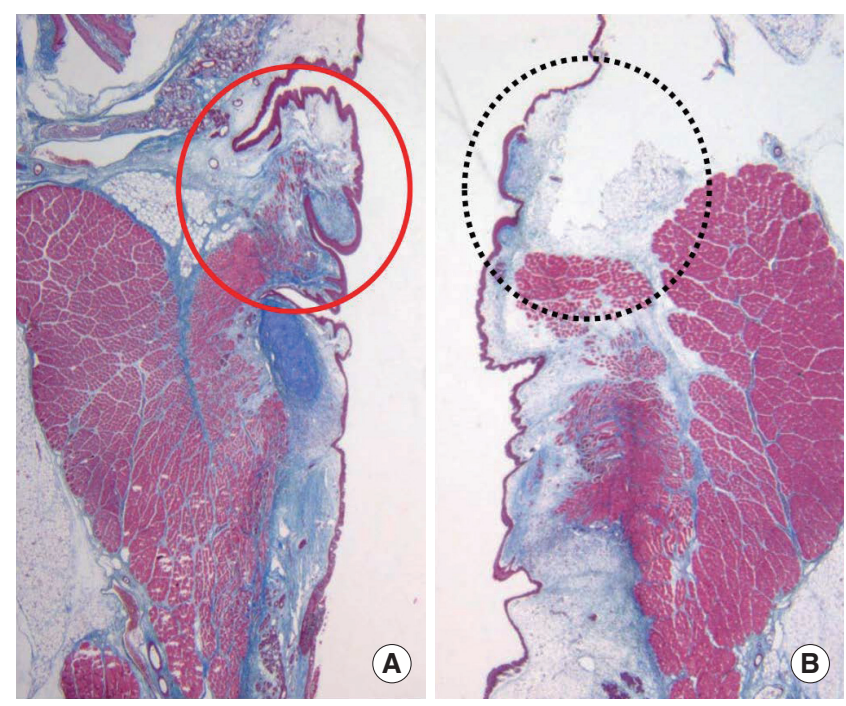

Fig. 4. Masson's trichrome staining of the vocal folds injected with $(A)$ phosphate-buffered saline (PBS; $0.1 \mathrm{~mL}$ ) and (B) fibroblast growth factor (FGF; $10 \mu \mathrm{g} / 0.1 \mathrm{~mL}$ ). Injection was performed 3 months after the scar-inducing injury. Coronal sections (6- $\mu \mathrm{m}$ thick) were prepared from the laryngeal tissues of 12 rabbits euthanized 6 months after scar formation. The FGF-injected fold (black dotted circle) shows greater vocal fold volume without an increase in the density of blue stained collagen $(\times 20)$ than PBS-injected fold (red circle). 
tion showed less stained blue-colored collagen in the lamina propria of the vocal fold (Fig. 4). The results showed that the relative density of collagen was significantly less on the FGF-injected than on the PBS-injected control group $(P<0.05)$ (Fig. 5); the relative density of HA was slightly, but not significantly, higher on the FGF-injected group.

\section{Voice analysis data after FGF injection in patients with chronic vocal fold scarring}

The mean volume and frequency of FGF injection was $0.25 \pm$ $0.12 \mathrm{~mL}$ and $1.85 \pm 0.6$ times, respectively. The mean duration until the achievement of subjective voice improvement after FGF injection was $47.9 \pm 25.6$ days. The demographic data of the patients are shown in Table 2. Voice parameters were compared with the baseline voice data at 3, 6, and 12 months postinjection. Except for the HNR, all objective parameters at 3 months post-injection had improved significantly. However, at 12 months post-injection, only trends towards an improvement in the percentage of jitter and shimmer had occurred. By con-

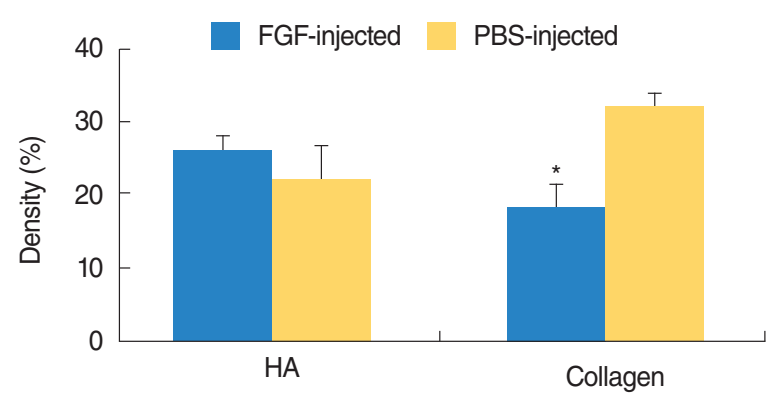

Fig. 5. Histological image analysis of hyaluronic acid (HA) and relative collagen density in the FGF-injected group vs. the PBS-injected group. The relative density of collagen was significantly lower whereas that of HA was not significantly higher in the FGF-injected group vs. PBS-injected groups. FGF, fibroblast growth factor; PBS, phosphate-buffered saline. ${ }^{*} P<0.05$ in a Mann-Whitney U-test. trast, the significant improvements in both the MPT and the VRP were retained at 12 months. All subjective parameters (GRABAS score, VHI-10, glottic closure grade, mucosal wave grade) became better at 3 months significantly and became steady at 12 months post-injection (Table 3). Stroboscopic findings and diagrams of the MDVP from a representative case are demonstrated (Fig. 6).

\section{Postoperative complication}

No serious adverse events, such as vocal fold granuloma formation, tumor growth, or vocal fold scar aggravation, occurred during follow-up period.

\section{DISCUSSION}

Vocal fold scarring damages the vibratory function of the vocal fold, resulting in serious voice problems, with particularly high social costs for individuals whose jobs rely on a healthy voice, such as teachers, performing artists, clergy, and telemarketers $[1,9]$. Moreover, voice disorders caused by vocal fold scarring are considered to be incurable, as current therapies, including surgical treatment (medialization thyroplasty, fat/collagen or ste-

Table 2. Demographic data of patients $(n=17)$

\begin{tabular}{lc}
\hline Characteristic & Value \\
\hline Sex (male:female) & $4: 13$ \\
Age (yr) & $50.35 \pm 15.12$ \\
No. of injections & $1.85 \pm 0.60$ \\
Volume of injection (mL) & $0.25 \pm 0.12$ \\
Follow-up period until improvement (day) & $47.94 \pm 45.60$ \\
Satisfaction score, VAS & $7.06 \pm 1.78$ \\
\hline
\end{tabular}

Values are presented as number or mean \pm standard deviation. VAS, visual analogue scale (1, extremely dissatisfied; 10, extremely satisfied).

Table 3. Voice analysis data of patients injected with fibroblast growth factor (FGF) to treat vocal fold scarring $(n=17)$

\begin{tabular}{|c|c|c|c|c|}
\hline \multirow{2}{*}{ Characteristic } & \multirow{2}{*}{ Baseline (SD) } & \multicolumn{3}{|c|}{ Duration after FGF injection (SD) } \\
\hline & & $3 \mathrm{Mo}$ & $6 \mathrm{Mo}$ & $12 \mathrm{Mo}$ \\
\hline Jitter (\%) & $3.19 \pm 2.30$ & $1.97 \pm 2.06^{\mathrm{a})}$ & $2.39 \pm 3.04$ & $1.85 \pm 2.22$ \\
\hline Shimmer (\%) & $8.27 \pm 4.45$ & $3.87 \pm 3.62^{a)}$ & $6.36 \pm 3.71$ & $7.14 \pm 7.57$ \\
\hline $\mathrm{NHR}(\%)$ & $0.22 \pm 0.10$ & $0.26 \pm 0.19$ & $0.19 \pm 0.14$ & $0.22 \pm 0.17$ \\
\hline MPT (sec) & $8.18(2.67)$ & $9.67(2.64)^{a)}$ & $10.30(1.00)^{\mathrm{b})}$ & $10.80(0.50)^{c)}$ \\
\hline $\operatorname{VR}(\mathrm{Hz})$ & $197.93(60.21)$ & $325.05(81.35)^{a)}$ & $278.90(38.55)^{b)}$ & $297.05(66.06)^{c)}$ \\
\hline GRABAS & $6.35(2.45)$ & $1.76(1.15)^{a)}$ & $1.71(1.25)^{\mathrm{b})}$ & $1.33(1.03)^{c)}$ \\
\hline VHI-10 & $21.94(8.47)$ & $12.29(8.15)^{a)}$ & $12.71(9.34)^{b)}$ & $3.17(2.64)^{c)}$ \\
\hline Glottic closure & $1.12(0.60)$ & $2.87(0.35)^{a}$ & $2.71(0.49)^{b)}$ & $2.80(0.45)^{c)}$ \\
\hline Mucosal wave & $1.12(0.60)$ & $2.40(0.63)^{a)}$ & $2.29(0.95)^{b)}$ & $2.40(0.55)^{c)}$ \\
\hline
\end{tabular}

Baseline, baseline voice data; SD, standard deviation; NHR, noise-to-harmonics ratio; MPT, maximum phonation time; VR, voice range profile; GRABAS, sum of grade, roughness, breathiness, asthenia, strain scale; VHI, Korean version of Voice Handicap Index-10; glottic closure, grades of glottis closure; mucosal wave, grades of glottic mucosal wave.

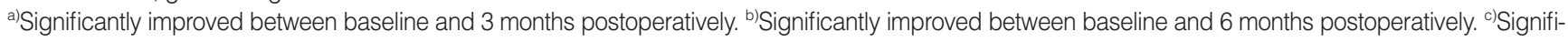
cantly improved between baseline and 12 months postoperatively. 

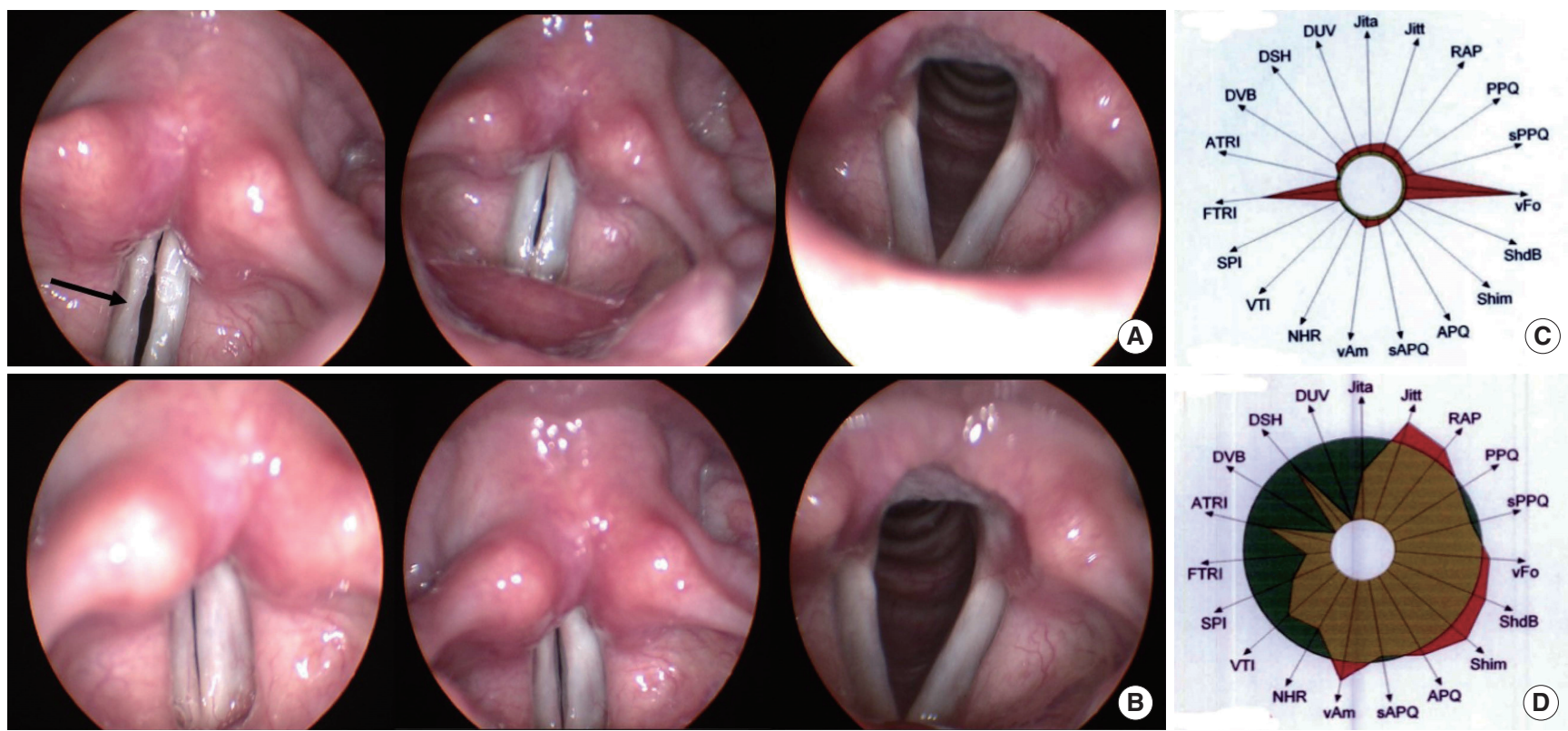

Fig. 6. Representative stroboscopic findings and Multi-Dimensional Voice Program (MDVP) diagrams of a patient who received fibroblast growth factor (FGF) injection. (A) Preoperative findings, demonstrating a chronic vocal focal scar with minimal glottis gap on the right vocal fold that impairs mucosal vibration (Glottal closure grade 2, Mucosal wave grade 1). The arrow highlights the scar. (B) 12 Months after injection. Vocal scar disappeared resulting in complete glottal closure and improved glottal vibratory function (Glottal closure grade 3, Mucosal wave grade 3). Glottal closure and the mucosal wave were graded on a four-point scale. $0=$ severe glottis gap, no wave; $1=$ moderate glottis gap, obvious decreased mucosal wave; 2 =mild glottis gap, slightly decreased mucosal wave; and 3=complete closure, full wave. Before (C) and after (D) FGF injection, the MDVP diagram showed improved objective voice parameters including Jitt, Shim, and NHR. Jita, absolute jitter $(\mu s)$; Jitt, jitter percent (\%); RAP, relative average perturbation (\%); PPQ, pitch perturbation quotient (\%); sPPQ, smoothed pitch perturbation quotient (\%); vFO, fundamental frequency variation (\%); ShdB, shimmer in dB (dB); Shim, shimmer percent (\%); APQ, amplitude perturbation quotient (\%); sAPQ, smoothed amplitude perturbation quotient (\%); vAm, peak-amplitude variation (\%); NHR, noise to harmonic ratio; VTI, Voice Turbulence Index; SPI, Soft Phonation Index; FTRI, FO-Tremor Intensity Index (\%); ATRI, Amplitude Tremor Intensity Index (\%); DVB, degree of voice breaks (\%); DSH, degree of sub-harmonics (\%); DUV, degree of voiceless (\%).

roid injection, and scar lysis) cannot restore the distorted layered structure of the vocal fold $[10,11]$.

Consequently, new tissue-engineering-based approaches, such as cell therapy [12], as well as the use of growth factors [13-17] are being pursued to treat vocal fold scarring. Hepatocyte growth factor has been previously reported for the treatment of chronic vocal fold scarring but its difficult manufacturing process has led to limited distribution. Another growth factor includes FGF, which plays a role in cell migration, angiogenesis, and the growth and differentiation of several tissue types. Fiblast (Kaken Pharmaceutical Co.), the form of FGF used in this study, is a commercialized spray of human recombinant bFGF. Repeated injection of this product to chronic vocal fold scar was clinically convenient and an additional drug delivery system, which can be restrictive in clinical use, was not necessary. Although commercial FGF application has been approved for treatment of skin ulcers, Hirano et al. [18] carried out a clinical study examining the effects of FGF in age-related vocal fold degeneration, based on the results of an aged rat model in which the ability of FGF to restore normal ECM synthesis by fibroblasts, reduce collagen type 1 mRNA expression in gingival fibroblasts, and stimulate HAS 2 synthesis by skin fibroblasts was demonstrated $[19,20]$. However, previous studies regarding the clinical application of FGF injections to vocal fold scars have been limited due to the small number of subjects and short clinical follow-up periods of less than 1 year to prove the potential safety. Hirano et al. [18] reported that repeated injections of bFGF ( $10 \mu \mathrm{g}$ in $0.5 \mathrm{~mL}$ saline) had a therapeutic effect in the vocal fold scar of 7 patients and Kanazawa et al. [21] demonstrated satisfactory voice quality 2 to 3 months after only a single injection of $50 \mu \mathrm{g}$ of bFGF in 0.5 $\mathrm{mL}$ saline into vocal fold of 6 patients. According to a previously reported injected dose of FGF, we injected $20 \mu \mathrm{g}$ of FGF in 0.2 $\mathrm{mL}$ reconstituted solution supplied by a Fiblast vial into the lesioned side of the human vocal fold. Before the clinical trial, 10 $\mu \mathrm{g}$ of FGF in $0.1 \mathrm{~mL}$ reconstituted solution, which is a similar concentration and total dose, was tried with bilateral rabbit vocal folds to confirm safety. Finally, we developed a rabbit model of chronic vocal scarring and then confirmed the restorative effect and safety of FGF in a larger clinical trial for long enough time about 1 year [20,21].

In our rabbit model of chronic vocal fold scarring, FGF injection decreased the relative density of collagen and increased 
mRNA level of HAS 2 and MMP 2 significantly. Increased collagen deposition and decreased HA are known as the most common histological findings in a scarred vocal cord. Both reflect genetic changes; specifically, an increase in procollagen type 1 and a decrease in HAS 2 and MMP 2 expression [3,22]. A corresponding improvement in vocal fold scarring was seen histologically. The relative density alterations in the ECM of the lamina propria after FGF injection was also confirmed histologically and was consistent with the significant regenerative effect of FGF vs. the PBS control. The regenerative change was also validated at the genetic level, by real-time PCR measurements of ECM gene expression in the FGF-injected vocal fold tissue. Although the density and mRNA level of HA and collagen were not correlated, we interpreted that the result was due to the difference in when the vocal fold was harvested, small number of included subjects in the experimental group $(n=6)$, and small volume of the rabbit vocal fold. Three months in the life of a rabbit is similar to several years in humans; therefore, our animal study based on 3 months of FGF injection was able to demonstrate the long-term regenerative effect of FGF. In our clinical prospective human clinical trial, patients who received FGF injections to treat chronic vocal fold scarring experienced significant improvements in both subjective and objective voice parameters for as long as 1 year, without severe side effects. The present study is not sufficient to conclude the efficacy of the FGF, because a control group, such as a PBS injection group in an animal model, is not provided. Further randomized control study is recommended to identify consistent efficacy.

In conclusion, based on a rabbit model of vocal fold scarring and the results of a prospective clinical trial, our study demonstrated that mild chronic vocal fold scarring can be successfully treated by FGF injection. The treated patients showed significantly improved voice quality for as long as 1 year, without side effects. Thus, our study recommends the use of FGF vocal fold injection as an alternative treatment modality in patients with mild chronic vocal fold scarring.

\section{CONFLICT OF INTEREST}

No potential conflict of interest relevant to this article was reported.

\section{ACKNOWLEDGMENTS}

This research was supported by the Soonchunhyang University Research Fund (support report no. 20130628).

\section{REFERENCES}

1. Hirano S. Current treatment of vocal fold scarring. Curr Opin Otolaryngol Head Neck Surg. 2005 Jun;13(3):143-7.

2. Suehiro A, Hirano S, Kishimoto Y, Rousseau B, Nakamura T, Ito J. Treatment of acute vocal fold scar with local injection of basic fibroblast growth factor: a canine study. Acta Otolaryngol. 2010 Jul;130 (7):844-50.

3. Rousseau B, Hirano S, Chan RW, Welham NV, Thibeault SL, Ford $\mathrm{CN}$, et al. Characterization of chronic vocal fold scarring in a rabbit model. JVoice. 2004 Mar;18(1):116-24.

4. Lim JY, Choi BH, Lee S, Jang YH, Choi JS, Kim YM. Regulation of wound healing by granulocyte-macrophage colony-stimulating factor after vocal fold injury. PLoS One. 2013;8(1):e54256.

5. Lee SW, Kim JW, Lee JY, Chang HS, Kim HK, Choi EC. Augmentation of vocal fold using a fat block implant following cordotomy through a minithyrotomy approach in a rabbit model. J Voice. 2012 Jul;26(4):521-5.

6. Hirano M. Clinical examination of voice.Wien: Springer-Verlag; 1981.

7. Yun YS, Kim HH, Son YI, Choi HS. Validation of the Korean Voice Handicap Index (K-VHI) and the clinical usefulness of Korean VHI10. Korean J Commun Disord. 2008 Jun;13(2):216-41.

8. Lee SW, Kim JW, Koh YW, Shim SS, Son YI. Comparative analysis of efficiency of injection laryngoplasty technique for with or without neck treatment patients: a transcartilaginous approach versus the cricothyroid approach. Clin Exp Otorhinolaryngol. 2010 Mar;3(1): 37-41.

9. Yoon KC, Mun GH, Kim SD, Kim SH, Kim CY, Park KH, et al. Prevalence of eye diseases in South Korea: data from the Korea National Health and Nutrition Examination Survey 2008-2009. Korean J Ophthalmol. 2011 Dec;25(6):421-33.

10. Hirano S, Minamiguchi S, Yamashita M, Ohno T, Kanemaru S, Kitamura M. Histologic characterization of human scarred vocal folds. J Voice. 2009 Jul;23(4):399-407.

11. Dailey SH, Ford CN. Surgical management of sulcus vocalis and vocal fold scarring. Otolaryngol Clin North Am. 2006 Feb;39(1):23-42.

12. Kanemaru S, Nakamura T, Omori K, Kojima H, Magrufov A, Hiratsuka Y, et al. Regeneration of the vocal fold using autologous mesenchymal stem cells. Ann Otol Rhinol Laryngol. 2003 Nov;112(11): 915-20.

13. Hirano S, Bless D, Heisey D, Ford C. Roles of hepatocyte growth factor and transforming growth factor beta1 in production of extracellular matrix by canine vocal fold fibroblasts. Laryngoscope. 2003 Jan;113(1):144-8.

14. Hirano S, Bless DM, Heisey D, Ford CN. Effect of growth factors on hyaluronan production by canine vocal fold fibroblasts. Ann Otol Rhinol Laryngol. 2003 Jul;112(7):617-24.

15. Hirano S, Bless DM, Rousseau B, Welham N, Montequin D, Chan RW, et al. Prevention of vocal fold scarring by topical injection of hepatocyte growth factor in a rabbit model. Laryngoscope. 2004 Mar;114(3): 548-56.

16. Hirano S, Bless DM, Nagai H, Rousseau B, Welham NV, Montequin DW, et al. Growth factor therapy for vocal fold scarring in a canine model.Ann Otol Rhinol Laryngol. 2004 Oct;113(10):777-85.

17. Ohno T, Hirano S, Kanemaru S, Yamashita M, Umeda H, Suehiro A, et al. Drug delivery system of hepatocyte growth factor for the treatment of vocal fold scarring in a canine model. Ann Otol Rhinol Laryngol. 2007 Oct;116(10):762-9.

18. Hirano S, Mizuta M, Kaneko M,Tateya I, Kanemaru SI, Ito J. Regenerative phonosurgical treatments for vocal fold scar and sulcus with basic fibroblast growth factor. Laryngoscope. 2013 Nov;123(11): 2749-55.

19. Hong HH, Trackman PC. Cytokine regulation of gingival fibroblast 
lysyl oxidase, collagen, and elastin. J Periodontol. 2002 Feb;73(2): 145-52.

20. Heldin P, Laurent TC, Heldin CH. Effect of growth factors on hyaluronan synthesis in cultured human fibroblasts. Biochem J. 1989 Mar;258(3):919-22.

21. Kanazawa T, Komazawa D, Indo K, Akagi Y, Lee Y, Nakamura K, et al. Single injection of basic fibroblast growth factor to treat severe vocal fold lesions and vocal fold paralysis. Laryngoscope. 2015 Oct; 125(1):E338-44.

22. Tateya T, Tateya I, Bless DM. Immuno-scanning electron microscopy of collagen types I and III in human vocal fold lamina propria. Ann Otol Rhinol Laryngol. 2007 Feb;116(2):156-9. 